\title{
Flow of viscoelastic fluids past a cylinder at high Weissenberg number: stabilized simulations using matrix logarithms
}

\author{
Martien A. Hulsen*, \\ Materials Technology, Eindhoven University of Technology, P.O. Box 513, \\ $5600 M B$, Eindhoven, The Netherlands \\ Raanan Fattal
}

School of Computer Science and Engineering, The Hebrew University, Jerusalem, 91904, Israel

Raz Kupferman

Institute of Mathematics, The Hebrew University, Jerusalem, 91904, Israel

\begin{abstract}
The log conformation representation proposed in [1] has been implemented in a FEM context using the DEVSS/DG formulation for viscoelastic fluid flow. We present a stability analysis in 1D and attribute the high Weissenberg problem to the failure of the numerical scheme to balance exponential growth. A slightly different derivation of the log based evolution equation than in [1] is also presented. We show numerical results for the flow around a cylinder for an Oldroyd-B and a Giesekus model. We provide evidence that the numerical instability identified in the $1 \mathrm{D}$ problem is also the actual reason for the failure of the standard FEM implementation of the problem. With the log conformation representation we are able to obtain solutions far beyond the limiting Weissenberg numbers in the standard scheme. However it turns out that, although in large parts of the flow the solution converges, we have not been able to obtain convergence in localized regions of the flow. Possible reasons for this are: artefacts of the model (Oldroyd-B) or unresolved small scales (Giesekus). However, more work is necessary, including the use of more refined meshes and/or higher-order schemes, before any conclusion can be made on the local convergence problems.
\end{abstract}

Key words: finite element method, matrix logarithm, flow around a cylinder, viscoelastic flow simulation 


\section{Introduction}

The high Weissenberg number problem (HWNP) has been the major stumbling block in computational rheology for the last three decades. The term "HWNP" coins the empirical observation that all numerical methods break down when the Weissenberg number exceeds a critical range. The precise critical value at which computations break down varies with the problem, the method and the mesh used. It is now widely accepted that the HWNP arises when computations are not resolved well enough to capture the sharp stress gradient that form within the flow, yet, the precise mechanism that leads to the breakdown has remained somewhat of a mystery (see [2] and the references therein).

The mechanism responsible for the HWNP has been recently explained in [3]. In essence, it is a numerical instability caused by the failure to balance the exponential growth of the stress (due to deformation) with convection. Such a failure is common to all methods that approximate the stress by polynomial base functions (the choice of polynomial base function is explicit in finite elements and implicit in finite differences). The proposed remedy was a change a variables into new variables that scale logarithmically with the stress tensor. Specifically, the constitutive relations were reformulated as equations for the matrix logarithm of the conformation tensor, exploiting the fact that the latter is symmetric positive definite. Numerical experiments for a two-dimensional lid-driven cavity using a second-order finite difference scheme indicate that schemes based on the new logarithmic formulation are immune to the high Weissenberg breakdown. The "old" HWNP is rather replaced with a "new" problem: accurate computations at high Weissenberg require high resolution. To some extent, the situation may now be compared to classical CFD, where stable calculations can be performed at arbitrarily large Reynolds numbers, but accuracy is lost when resolution is insufficient.

In this paper we implement the new logarithmic formulation with a finite element method (FEM), and test it for a classical benchmark problem-flow past a cylinder. More specifically, we have in mind the following goals:

(1) Develop a FEM scheme immune to the high Weissenberg instability.

(2) Get more insight into the HWNP by analyzing the numerical breakdown within a FEM point of view.

(3) Present benchmark results that confirm the validity of the new method at low and moderate Weissenberg numbers, where comparison to existing data is possible.

\footnotetext{
* Corresponding author. Tel: +31-40-247-5081; Fax: +31-40-244-7355.

Email addresses: m.a.hulsen@tue.nl (Martien A. Hulsen), raananf@cs.huji.ac.il (Raanan Fattal), raz@math.huji.ac.il (Raz Kupferman).
} 
(4) Establish new benchmark results at higher Weissenberg numbers.

(5) Investigate the limitations of the method, and in particular, understand how accuracy is lost at high Weissenberg numbers. Such an understanding is important for the future design of higher-order methods.

The paper is structured as follows: In Section 2 we describe the governing equations and introduce the notations used henceforth. In Section 3 we describe the standard FEM discretization based on DEVSS and discontinuous Galerkin. In Section 4 we analyze the stability of the standard discretization, and in particular obtain a simple stability criterion whose violation causes the high Weissenberg breakdown. In Section 5 we derive the constitutive equation for the matrix logarithm of the conformation tensor; the derivation is based on a slightly different approach than in [1]. In Section 6 we present numerical results for the flow past a cylinder for an Oldroyd-B and a Giesekus model. In particular we will verify the criterion for breakdown in the standard discretization. We also show the much improved stability of the matrix log formulation. A discussion follows in Section 7.

\section{Governing equations}

We will consider the flow of viscoelastic fluids at creeping flow conditions (inertia can be neglected) in a spatial region $\Omega$, having a boundary denoted by $\Gamma$. For this, we need the following set of equations: the momentum balance, the mass balance for incompressible flows and a constitutive equation decribing the stress.

The momentum balance and mass balance are given by

$$
\begin{gathered}
\nabla p-\nabla \cdot\left(2 \eta_{\mathrm{s}} \boldsymbol{D}\right)-\nabla \cdot \boldsymbol{\tau}=\mathbf{0} \\
\nabla \cdot \boldsymbol{u}=0
\end{gathered}
$$

where $p$ is the pressure, $\boldsymbol{u}$ is the velocity vector, the rate-of-deformation tensor is given by $\boldsymbol{D}=\frac{1}{2}\left(\boldsymbol{L}+\boldsymbol{L}^{T}\right)$, with the velocity gradient tensor $\boldsymbol{L}=(\nabla \boldsymbol{u})^{T}$ and $\boldsymbol{\tau}$ is the extra-stress (or polymer stress). The coefficient $\eta_{\mathrm{s}}$ is the solvent viscosity. The polymer stress $\boldsymbol{\tau}$ is given by

$$
\boldsymbol{\tau}=\frac{\eta_{\mathrm{p}}}{\lambda}(\boldsymbol{c}-\boldsymbol{I})
$$

where $\boldsymbol{c}$ is the conformation tensor, $\eta_{\mathrm{p}}$ is the zero-shear-rate viscosity of the polymer part of the stress and $\lambda$ is the relaxation time. We will use two different models for the evolution of the conformation tensor: the Oldroyd-B and the Giesekus model, which can be written as follows

$$
\dot{c}=\boldsymbol{L} \cdot \boldsymbol{c}+\boldsymbol{c} \cdot \boldsymbol{L}^{T}+\boldsymbol{f}(\boldsymbol{c}),
$$


with

$$
\boldsymbol{f}(\boldsymbol{c})= \begin{cases}-\frac{1}{\lambda}(\boldsymbol{c}-\boldsymbol{I}) & \text { Oldroyd-B, } \\ -\frac{1}{\lambda}\left(\boldsymbol{c}-\boldsymbol{I}+\alpha(\boldsymbol{c}-\boldsymbol{I})^{2}\right) & \text { Giesekus. }\end{cases}
$$

The Oldroyd-B model is identical to a Giesekus model with $\alpha=0$. We will solve Eq. (4) in a Eulerian frame and therefore write

$$
\dot{\boldsymbol{c}}=\frac{\partial \boldsymbol{c}}{\partial t}+\boldsymbol{u} \cdot \nabla \boldsymbol{c}
$$

Boundary conditions will be discussed for the flow around a cylinder problem that will be analysed in Sec. 6 .

\section{Numerical discretization}

For the spatial discretisation of the system of equations we will use the finite element method (FEM). We will basically use the same implementation as described in [4] and give a brief summary here.

In order to obtain a proper mixed velocity-stress formulation we use the Discrete Elastic-Viscous Split Stress (DEVSS) formulation of Guénette \& Fortin [5] for the discretisation of the linear momentum balance and the continuity equation. For this we introduce an extra variable $\boldsymbol{e}=2 \eta_{\mathrm{p}} \boldsymbol{D}$. Note, that Guénette \& Fortin [5] introduce $\boldsymbol{D}$ as an extra variable, however using $\boldsymbol{e}$ instead leads to a symmetric system matrix. We rewrite the momentum balance Eq. (1) and the continuity equation Eq. (2) as follows

$$
\begin{gathered}
\nabla p-\nabla \cdot\left(2 \eta_{\mathrm{s}} \boldsymbol{D}(\boldsymbol{u})+\boldsymbol{\tau}\right)-\nabla \cdot\left(2 \eta_{\mathrm{p}} \boldsymbol{D}(\boldsymbol{u})-\boldsymbol{e}\right)=\mathbf{0} \\
-\nabla \cdot \boldsymbol{u}=0, \\
-\boldsymbol{D}(\boldsymbol{u})+\frac{1}{2 \eta_{\mathrm{p}}} \boldsymbol{e}=\mathbf{0} .
\end{gathered}
$$

For the weak formulation of Eqs. (7)-(9) we introduce separate functional spaces for $\boldsymbol{u}, p$ and $\boldsymbol{e}$, which we denote by $U, P$ and $E$, respectively. The weak formulation can be found by multiplying with testfunctions and integration by parts: find $(\boldsymbol{u}, p, \boldsymbol{e}) \in U \times P \times E$ such that for all $(\boldsymbol{v}, q, \boldsymbol{g}) \in U \times P \times E$ we have

$$
\begin{gathered}
-(\nabla \cdot \boldsymbol{v}, p)+(\nabla \boldsymbol{v}, 2 \eta \boldsymbol{D}(\boldsymbol{u})-\boldsymbol{e}+\boldsymbol{\tau})=(\boldsymbol{v}, \boldsymbol{\sigma})_{\Gamma}, \\
-(q, \nabla \cdot \boldsymbol{u})=0, \\
-(\boldsymbol{g}, \nabla \boldsymbol{u})+\frac{1}{2 \eta_{\mathrm{p}}}(\boldsymbol{g}, \boldsymbol{e})=0,
\end{gathered}
$$


where $(\cdot, \cdot)$ and $(\cdot, \cdot)_{\Gamma}$ are proper $L^{2}$ inner products on the domain $\Omega$ and on the boundary $\Gamma$, respectively. The viscosity $\eta$ is the zero-shear-rate viscosity $\eta_{\mathrm{s}}+\eta_{\mathrm{p}}$ and $\boldsymbol{\sigma}$ is the traction vector on the boundary. The system for $(\boldsymbol{u}, p, \boldsymbol{e})$ is symmetrical.

The discrete form of the equations is obtained by requiring that the weak form is valid on approximating subspaces $U_{h} \times P_{h} \times E_{h}$ which consist of piecewise polynomial spaces. The discrete solutions and the discrete testfunctions are denoted with subindex ${ }_{h}:\left(\boldsymbol{u}_{h}, p_{h}, \boldsymbol{e}_{h}\right)$ and $\left(\boldsymbol{v}_{h}, q_{h}, \boldsymbol{g}_{h}\right)$. We will use quadrilateral elements with continuous biquadratic polynomials $\left(Q_{2}\right)$ for the velocity space $U_{h}$, discontinuous linear polynomials $\left(P_{1}\right)$ for the pressure space $P_{h}$ and continuous bilinear polynomials $\left(Q_{1}\right)$ for the viscous polymer stress space $E_{h}$.

For the discretisation of the constitutive equation we use the discontinuous Galerkin method (DG) [6]. This leads to the following weak formulation of the constitutive equation Eq. (4): find $c \in T$ on all elements $e_{i}$ such that for all $\boldsymbol{w} \in T$ we have

$$
\begin{aligned}
\left(\boldsymbol{w}, \frac{\partial \boldsymbol{c}}{\partial t}+\boldsymbol{u} \cdot \nabla \boldsymbol{c}-\boldsymbol{L} \cdot \boldsymbol{c}-\boldsymbol{c} \cdot \boldsymbol{L}^{T}-\right. & \boldsymbol{f}(\boldsymbol{c}))_{e_{i}} \\
& +\int_{\gamma_{i}^{\text {in }}}(\boldsymbol{n} \cdot \boldsymbol{u}) \boldsymbol{w}:\left(\boldsymbol{c}^{+}-\boldsymbol{c}\right) \mathrm{d} \gamma=0,
\end{aligned}
$$

where $(\cdot, \cdot)_{e_{i}}$ denotes an $L^{2}$ inner product on element $e_{i}$ only, $\gamma_{i}^{\text {in }}$ is the part of the element boundary where $\boldsymbol{u} \cdot \boldsymbol{n}<0$ (inflow boundary), $\boldsymbol{n}$ is the outwardly directed unit normal on $\gamma_{i}^{\text {in }}$. Furthermore, $\boldsymbol{c}^{+}$is the value of $\boldsymbol{c}$ in the upstream neighbour element or the imposed value at the inflow boundary part of $\Gamma$. The functional space for $\boldsymbol{c}$ is denoted by $T$. We will use discontinuous bilinear polynomials $\left(Q_{1}\right)$ for the space $T_{h}$. The combination of the DEVSS formulation with DG has been introduced by Baaijens et al. [7] and it has been proved by Fortin et al. [8] that it leads to a proper mixed velocity-stress scheme.

For the time discretisation of the constitutive equation Eq. (13) we use an explicit Euler scheme, where the time derivative is discretised by $\partial \boldsymbol{c} / \partial t \approx$ $\left.\left(\boldsymbol{c}^{n+1}-\boldsymbol{c}^{n}\right)\right) / \Delta t$ and all other terms are evaluated at $t_{n}$. For time-accurate solutions we can use a higher-order scheme, such as Adams-Bashforth, but for obtaining steady state solutions this is not necessary. Note, that the equations can be solved at element level. Next we substitute $\boldsymbol{\tau}^{n+1}=\eta_{\mathrm{p}} / \lambda\left(\boldsymbol{c}^{n+1}-\boldsymbol{I}\right)$ into Eq. (10). The system matrix for solving $\left(\boldsymbol{u}_{n+1}, p_{n+1}, \boldsymbol{e}_{n+1}\right)$ is symmetrical and $\mathrm{LU}$ decomposition is performed at the first time step. Since this matrix is constant in time, solutions at later time steps can be found by back substitution only. This results in a significant reduction of the CPU time. 


\section{A stability criterion for exponential profiles}

In [3] an analysis of the HWNP is given for a one-dimensional problem discretized using a first-order upwind scheme. Here we shortly repeat that and at the same time extend the analysis to our DG scheme.

The 'toy problem' we are considering here is as follows: find $c(x, t)$ with $x$ in the interval $(0, L)$ and time $t>0$ such that

$$
\frac{\partial c}{\partial t}+a \frac{\partial c}{\partial x}=b c
$$

where $a$ is a constant convection speed and $b>0$ is the exponential growth factor. Note, that Eq. (14) can be interpreted as a 1D representation of the model for the conformation tensor Eq. (4) in the Eulerian frame (using Eq. (6)) with $a \sim \boldsymbol{u}$ and $b \sim \boldsymbol{L}-1 / \lambda$ (see below). For simplicity we assume $a>0$ for now. We will assume $c(x, t=0)=1$ as initial condition and $c(x=0, t)=1$ as inflow condition. The analytical solution is given by

$$
c(x, t)= \begin{cases}\exp \left(\frac{b x}{a}\right) & \text { for } x \leq a t, \\ \exp (b t) & \text { for } a t<x \leq L .\end{cases}
$$

The solution is split into a region where it is steady but exponential growing in space with a growth factor $b / a$ and a region where the solution is exponential growing in time with a growth factor of $b$. At time $t=L / a$ the time-dependent region disappears and the solution is steady for $t>L / a$. Despite the exponential growth factor $b$, it is possible to obtain a steady state due to balancing of exponential growth by the convection.

Now assume a grid having $N$ equidistant intervals of size $\Delta x$. The coordinates are $x_{i}=i \Delta x, i=0, \ldots, N$. If we discretize Eq. (14) in space using a first-order upwind scheme [9] and incorporate the inflow condition we get

$$
\begin{aligned}
\frac{\mathrm{d} c_{i}}{\mathrm{~d} t} & =-a \frac{c_{i}-c_{i-1}}{\Delta x}+b c_{i} \\
& =\left(b-\frac{a}{\Delta x}\right) c_{i}+\frac{a}{\Delta x} c_{i-1}, \quad i=1, \ldots N
\end{aligned}
$$

with $c_{i}(t)=c\left(x_{i}, t\right)$ and $c_{0}=1$. Assuming that both $c_{i}$ and $c_{i-1}$ are positive it is easy to see that the coefficient of $c_{i}$ must be negative:

$$
b-\frac{a}{\Delta x}<0 \quad \text { or } \quad \Delta x<\frac{a}{b}=\Delta x_{\text {crit }},
$$

otherwise $\mathrm{d} c_{i} / \mathrm{d} t$ remains positive and the numerical solution is not able to obtain a steady state, as the exact solution does. The source of the numerical 
instability is the failure to balance the exponential growth by convection. We believe this is the cause of the HWNP. Note, that in the grid points of the steady region the exact solution Eq. (15) can be written as

$$
c_{i}=\exp \left(\frac{b x_{i}}{a}\right)=\left(\exp \left(\frac{\Delta x}{\Delta x_{\text {crit }}}\right)\right)^{i} .
$$

Using this, we can interpret the stability criterion Eq. (17) as saying that $\Delta x$ must be such that the growth factor in a single cell must be smaller that $e$.

In order to derive a similar stability criterion for DG we rewrite Eq. (16) into a matrix system:

$$
\frac{\mathrm{d} c}{\mathrm{~d} t}=\left(\begin{array}{cccc}
\gamma & & & 0 \\
\beta & \gamma & & \\
& \ddots & \ddots & \\
0 & & & \beta \gamma
\end{array}\right) c+f, \quad \text { with } \gamma=b-\frac{a}{\Delta x}, \quad \beta=\frac{a}{\Delta x}
$$

For obtaining a steady numerical solution it is required that the eigenvalue $(=\gamma)$ of the coefficient matrix must be negative. This is identical to Eq. (17). For DG with linear polynomial interpolation we can write the discretized equations in the same form as Eq. (19), however now $\gamma$ and $\beta$ are $2 \times 2$ matrices (see [10]):

$$
\gamma=b\left(\begin{array}{ll}
1 & 0 \\
0 & 1
\end{array}\right)+\frac{a}{\Delta x}\left(\begin{array}{cc}
-3 & -1 \\
3 & -1
\end{array}\right), \quad \beta=\frac{a}{\Delta x}\left(\begin{array}{cc}
0 & 4 \\
0 & -2
\end{array}\right) .
$$

The eigenvalues of the coefficient matrix are identical to the eigenvalues of $\gamma$, which are $b+(-2 \pm i \sqrt{2}) a / \Delta x$. For stability the real part of the eigenvalues must be negative:

$$
b-2 \frac{a}{\Delta x}<0 \quad \text { or } \quad \Delta x<2 \frac{a}{b} .
$$

Now let's introduce a dimensionless grid number $C$ :

$$
C=\Delta x \frac{b}{|a|}
$$

then Eqs. (17) and (21) can now be written as

$$
\begin{array}{ll}
C<1 & \text { for first-order upwind, } \\
C<2 & \text { for DG with } P_{1} \text { interpolation, }
\end{array}
$$

respectively. Note, that we have extended the definition of $C$ such that both positive and negative values of $a$ are allowed. 
As explained in [1] and [3] the numerical instability described above can be removed by solving for $s=\log c$ instead of $c$. This removes exponential profiles from the quantity that is being solved and therefore also the source of the instability. The exponential growth is fully contained within the variable $s$ and only becomes visible when tranforming back with $c=\exp (s)$. The equation for $s$ can easily be derived, using $\dot{s}=\dot{c} / c$ with $\dot{s}=\partial s / \partial t+a \partial s / \partial x$ :

$$
\frac{\partial s}{\partial t}+a \frac{\partial s}{\partial x}=b
$$

Note that the right-hand side is now a constant. The analytical solution for $s$ is

$$
s(x, t)= \begin{cases}\frac{b x}{a} & \text { for } x \leq a t, \\ b t & \text { for } a t<x \leq L,\end{cases}
$$

which clearly shows the linear behavior of $s$ both in the steady region and the time-dependent region.

A possible extension of the definition of $C$ to $2 \mathrm{D}$ is suggested by considering the discretization of

$$
\frac{\partial c}{\partial t}+a_{x} \frac{\partial c}{\partial x}+a_{y} \frac{\partial c}{\partial y}=b c
$$

on a regular grid with first-order upwinding. In that case, the coefficient of $c_{i}$ becomes $b-a_{x} / \Delta x-a_{y} / \Delta y$ and therefore

$$
C=\frac{b}{\frac{\left|a_{x}\right|}{\Delta x}+\frac{\left|a_{y}\right|}{\Delta y}},
$$

with $\Delta x$ and $\Delta y$ a typical size of the elements in $x$ and $y$ direction, respectively, seems to be a reasonable extension to 2D. Another possible extension is given in [3], where $a$ in Eq. (22) is replaced by $\left(a_{x}^{2}+a_{y}^{2}\right)^{\frac{1}{2}}$ and $\Delta x$ represents a typical grid size in the direction of the 'velocity' $\left(a_{x}, a_{y}\right)$.

Application of the analysis above to the viscoelastic constitutive model Eq. (4) is rather straightforward. The coefficients $a$ and $\left(a_{x}, a_{y}\right)$ represent the velocity vector $\boldsymbol{u}$, thus $a=u$ in $1 \mathrm{D}$ and $\left(a_{x}, a_{y}\right)=(u, v)$ in $2 \mathrm{D}$. The coefficient $b$ represents the positive growth rate of the right-hand side of Eq. (4). Since, the constitutive model is a tensor equation, we need to compute the eigenvalues of the coefficient matrix of the right-hand side. For the Giesekus model we first linearize the right-hand side and compute the eigenvalues of coefficient matrix of the perturbed system. The eigenvalues are derived in $[11,12]$ and the result shows that for two-dimensional flows a single eigenvalue becomes positive when

$$
\operatorname{det} \boldsymbol{C}<0, \quad \text { with } \boldsymbol{C}=-\frac{1}{2 \lambda}+\boldsymbol{L}-\frac{\alpha}{\lambda}(\boldsymbol{c}-\boldsymbol{I})
$$


The value of the positive eigenvalue $\mu_{\max }$ is given by

$$
\mu_{\max }=\operatorname{tr} \boldsymbol{C}+\left[(\operatorname{tr} \boldsymbol{C})^{2}-4 \operatorname{det} \boldsymbol{C}\right]^{\frac{1}{2}} .
$$

Hence, we have to set $b=\mu_{\max }$ when there is a positive eigenvalue. For negative or complex eigenvalues we just set $b=0$, making $C=0$. Note, that for the Oldroyd-B model $(\alpha=0)$, Eqs. (29) and (30) can be simplified to

$$
\mu_{\max }=-\frac{1}{\lambda}+2 \sqrt{-\operatorname{det} \boldsymbol{L}}, \quad \text { for } \operatorname{det} \boldsymbol{L}<-\frac{1}{4 \lambda^{2}},
$$

which is consistent with the stability criterium in [3] that is derived directly from the discretized equations. In planar extension the eigenvalues are all real and the Eqs. (29) and (30) combined with Eq. (22) can be written in the compact form

$$
C=\Delta x \frac{\max \left(2 \dot{\epsilon}-\frac{1}{\lambda}\left[1+2 \alpha\left(c_{x x}-1\right)\right], 0\right)}{|u|},
$$

where it has been assumed that $\dot{\epsilon}=L_{x x}>0$ (positive stretching).

The value of $C$ becomes critical in regions where we have a high stretching rate combined with a small velocity. Particularly troublesome are stagnation points and geometric singularities (sharp corners), which confirms the experience that problems containing these are the most difficult to simulate for higher Weissenberg numbers.

As suggested in [1] and [3] the numerical instability for viscoelastic flows can be removed, like in the 1D toy problem, by solving for $\boldsymbol{s}=\log \boldsymbol{c}$ instead of $\boldsymbol{c}$. This removes exponential profiles from the quantity that is being solved and therefore also the source of the instability. In the next section we will derive an evolution equation for $\boldsymbol{s}$ that will replace the evolution equation for $\boldsymbol{c}$.

\section{Evolution equation for the logarithm of the conformation tensor}

In the previous section we have identified the failure to resolve exponential profiles as a major restriction on the stability of standard schemes. It is suggested that this restriction can be lifted by solving for the matrix-logarithm of the conformation tensor $\boldsymbol{c}$ instead of $\boldsymbol{c}$ itself. In order to do so, we need an evolution equation for $\boldsymbol{s}=\log \boldsymbol{c}$. In [1] this evolution equation has been derived by a decomposition of the velocity gradient. In this paper to give an alternative derivation using an approach which is related to the work of Hill [13] on the evolution of the principal axes of the deformation tensor. 
The conformation tensor $\boldsymbol{c}$ is a symmetric positive definite tensor and therefore $\boldsymbol{s}=\log \boldsymbol{c}$ can be uniquely defined in terms of the principal direction $\boldsymbol{n}_{i}$, $i=1,2,3$ of $\boldsymbol{c}$ :

$$
\boldsymbol{s}=\log \boldsymbol{c}=\sum_{i=1}^{3} \log \left(c_{i}\right) \boldsymbol{n}_{i} \boldsymbol{n}_{i},
$$

or $s$ is coaxial with $\boldsymbol{c}$ and its principal values $s_{i}$ are given by $s_{i}=\log \left(c_{i}\right)$. The evolution of $\boldsymbol{s}$ can be computed from Eq. (33) as follows

$$
\dot{\boldsymbol{s}}=\sum_{i=1}^{3} \frac{\dot{c}_{i}}{c_{i}} \boldsymbol{n}_{i} \boldsymbol{n}_{i}+\sum_{i=1}^{3} s_{i} \dot{\boldsymbol{n}}_{i} \boldsymbol{n}_{i}+\sum_{i=1}^{3} s_{i} \boldsymbol{n}_{i} \dot{\boldsymbol{n}}_{i}
$$

So we need expressions for $\dot{c}_{i}$ and $\dot{\boldsymbol{n}}_{i}$. These can be determined from the constitutive equation, as we will show below. First we need to write $\dot{\boldsymbol{n}}_{i}$ in a different way.

The principal directions $\boldsymbol{n}_{i}$ are orthogonal vectors of a constant (unit) length. Therefore the time derivatives $\dot{\boldsymbol{n}}_{i}$ are given by just three independent quantities, which can be written in the form of an skew-symmetric tensor $\boldsymbol{\omega}$ :

$$
\dot{\boldsymbol{n}}_{i}=\boldsymbol{\omega} \cdot \boldsymbol{n}_{i}=\sum_{j=1}^{3} \omega_{j i} \boldsymbol{n}_{j}
$$

where $\omega_{i j}$ are the components of $\boldsymbol{\omega}$ with respect to the principal directions, i.e. $\omega_{i j}=\boldsymbol{n}_{i} \cdot \boldsymbol{\omega} \cdot \boldsymbol{n}_{j}$. Note that $\omega_{i j}=-\omega_{i j}$ for $i \neq j$ and $\omega_{i j}=0$ for $i=j$. Now Eq. (34) can be written as follows

$$
\begin{aligned}
\dot{\boldsymbol{s}} & =\sum_{i=1}^{3} \frac{\dot{c}_{i}}{c_{i}} \boldsymbol{n}_{i} \boldsymbol{n}_{i}+\boldsymbol{\omega} \cdot \boldsymbol{s}+\boldsymbol{s} \cdot \boldsymbol{\omega}^{T} \\
& =\sum_{i=1}^{3} \frac{\dot{c}_{i}}{c_{i}} \boldsymbol{n}_{i} \boldsymbol{n}_{i}+\sum_{i=1}^{3} \sum_{j=1}^{3}\left(s_{i}-s_{j}\right) \omega_{j i} \boldsymbol{n}_{i} \boldsymbol{n}_{j} .
\end{aligned}
$$

Note, that the last term is indeed a symmetric tensor.

Now we write $\dot{\boldsymbol{c}}$ in a similar way:

$$
\dot{\boldsymbol{c}}=\sum_{i=1}^{3} \dot{c}_{i} \boldsymbol{n}_{i} \boldsymbol{n}_{i}+\sum_{i=1}^{3} \sum_{j=1}^{3}\left(c_{i}-c_{j}\right) \omega_{j i} \boldsymbol{n}_{i} \boldsymbol{n}_{j} .
$$

and transform the right-hand side of the constitutive equation Eq. (4) to the principal directions:

$$
\dot{\boldsymbol{c}}=\boldsymbol{L} \cdot \boldsymbol{c}+\boldsymbol{c} \cdot \boldsymbol{L}^{T}+\boldsymbol{f}(\boldsymbol{c})=\sum_{i=1}^{3} \sum_{j=1}^{3}\left(c_{j} L_{i j}+c_{i} L_{j i}\right) \boldsymbol{n}_{i} \boldsymbol{n}_{j}+\sum_{i=1}^{3} f_{i}\left(c_{1}, c_{2}, c_{3}\right) \boldsymbol{n}_{i} \boldsymbol{n}_{i},
$$

where we have written $f_{i}$ explicitly as a function of the principal values of $\boldsymbol{c}$, using the property that $\boldsymbol{f}(\boldsymbol{c})$ is an isotropic function. Equating the diagonal 
components of Eqs. (37) and (38) leads to

$$
\dot{c}_{i}=2 c_{i} L_{i i}+f_{i}\left(c_{1}, c_{2}, c_{3}\right), \quad i=1,2,3
$$

and from the off-diagonal components we get:

$$
\omega_{i j}=\frac{c_{i} L_{j i}+c_{j} L_{i j}}{c_{j}-c_{i}}, \quad i, j=1,2,3, \quad i \neq j, \quad c_{i} \neq c_{j} .
$$

Substituting these results into Eq.(36) leads to our final result for the evolution of $\boldsymbol{s}=\log \boldsymbol{c}$ :

$$
\dot{\boldsymbol{s}}=\sum_{i=1}^{3}\left(2 L_{i i}+\frac{f_{i}}{c_{i}}\right) \boldsymbol{n}_{i} \boldsymbol{n}_{i}+\sum_{\substack{i=1 \\ i \neq j}}^{3} \sum_{j=1}^{3} \frac{s_{i}-s_{j}}{c_{i}-c_{j}}\left(c_{j} L_{i j}+c_{i} L_{j i}\right) \boldsymbol{n}_{i} \boldsymbol{n}_{j} .
$$

Note that:

- In the limit that two principal values are the same, we have:

$$
\lim _{c_{i} \rightarrow c_{j}} \frac{s_{i}-s_{j}}{c_{i}-c_{j}}\left(c_{j} L_{i j}+c_{i} L_{j i}\right)=L_{i j}+L_{j i}=2 D_{i j},
$$

and the $i j$ - and $j i$-components of the non-diagonal term in Eq. (41) nicely add-up to the diagonal term to form a tensor in the $\left(\boldsymbol{n}_{i}, \boldsymbol{n}_{j}\right)$ plane independent of the principal vectors, as it should, since these are not unique in this case. If all principal values are the same, the terms add up to the full tensor $2 D$.

- The term with $f_{i}$ can be written in tensorial form as follows:

$$
\sum_{i=1}^{3} \frac{f_{i}}{c_{i}} \boldsymbol{n}_{i} \boldsymbol{n}_{i}=\boldsymbol{c}^{-1} \cdot \boldsymbol{f}(\boldsymbol{c}) .
$$

- There is a clean separation of effects in Eq. (41) (see also Eq. (36)):

- stretching along the principal axes with $L_{i i}$,

- relaxation along the principal axes $\left(f_{i} / c_{i}\right)$,

- rotation of the principal axes with $\boldsymbol{\omega}$.

- Like in the 1D toy problem, the stretching term is additive rather than multiplicative.

- In the actual implementation it is easy to replace Eq. (4) by Eq. (41). Additional requirements are a routine that computes the principal directions and values of $\boldsymbol{s}$ and transformations of the components of a tensor in the global frame to the principal frame and vice versa. Furthermore, to compute the stress tensor $\boldsymbol{\tau}$, the conformation tensor $\boldsymbol{c}$ needs to be computed from $\boldsymbol{s}$, which is most easily performed in the principal frame using $c_{i}=\exp \left(s_{i}\right)$ and then a transform to the global frame.

- Since the conformation tensor $\boldsymbol{c}$ is computed from $\boldsymbol{s}$ using $\boldsymbol{c}=\exp \boldsymbol{s}$, it is positive definite 'by design', even if $s$ contains large numerical errors. This 


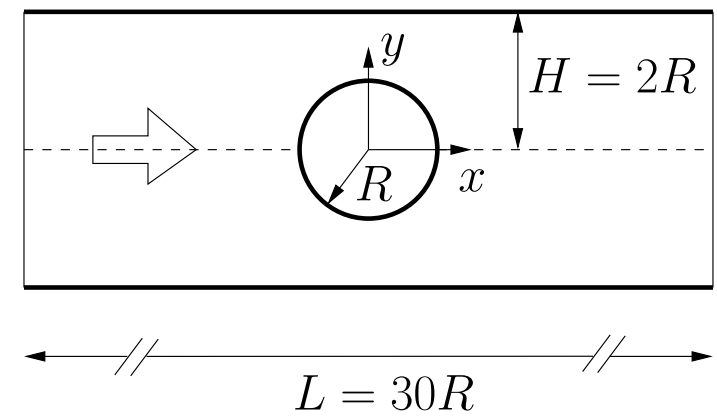

Fig. 1. Geometry of the cylinder between two flat plates. The flow is from left to right.

property probably also contributes to the stability of the log based scheme. This is similar to the situation with Brownian configuration fields [4], where the method always produces a positive definite conformation tensor.

- The resulting Eq. (41) is in agreement with [1].

\section{Results for the flow around cylinder confined between two plates}

\subsection{Problem description}

We consider the planar flow past a cylinder of radius $R$ positioned between two flat plates separated by a distance $2 H$. The ratio $R / H$ is equal to 2 and the total length of the flow domain is $30 R$. The flow geometry is shown in Fig. 1. In the following we will use an $(x, y)$ co-ordinate system with the origin positioned at the centre of the cylinder.

We assume the flow to be periodic. This means that we periodically extend the flow domain such that cylinders are positioned $30 R$ apart. This avoids specification of inflow and outflow boundary conditions. The flow is generated by specifying a flow rate $Q$ that is constant in time. The required pressure gradient is computed at each instant in time. We assume no-slip boundary conditions on the cylinder and on the walls of the channel. Since the problem is assumed to be symmetric we only consider half of the domain and use symmetry conditions on the centre line, i.e. zero tangential traction.

The dimensionless parameters governing the problem are the Weissenberg number $\mathrm{Wi}=\lambda U / R$ and the viscosity ratio $\eta_{\mathrm{s}} / \eta$ where $U=Q / 2 H$ is the average velocity and $\eta=\eta_{\mathrm{s}}+\eta_{\mathrm{p}}$ is the zero-shear-rate viscosity of the fluid. In this paper we use $\eta_{\mathrm{s}} / \eta=0.59$, which is the value used in benchmarks for the Oldroyd-B model.

In the following we will only use dimensionless quantities: the time variable 


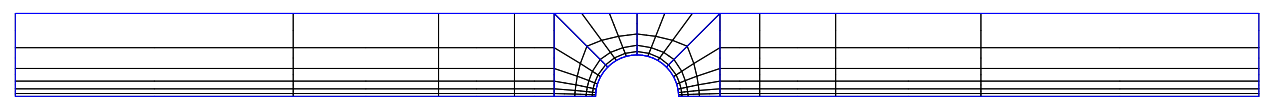

Fig. 2. Base mesh M0 from which all other meshes are derived. The base mesh M0 has 120 elements.

Table 1

Numerical parameters

\begin{tabular}{llllll}
\hline & M3 & M4 & M5 & M6 & M7 \\
\hline Number of elements $N_{\mathrm{el}}$ & 1920 & 4320 & 7680 & 17280 & 30720 \\
Number of nodal points & 7921 & 17461 & 31201 & 69841 & 123841 \\
Smallest radial element size & 0.0220 & 0.0148 & 0.0111 & 0.00743 & 0.00558 \\
smallest $\Delta t$ used & $5 \times 10^{-3}$ & $3 \times 10^{-3}$ & $3 \times 10^{-3}$ & $2 \times 10^{-3}$ & $1.5 \times 10^{-3}$
\end{tabular}

has been made dimensionless with the characteristic time scale of the flow $R / U$, velocities with $U$, lengths with $L$, stresses with $\eta U / R$.

To solve the problem numerically we used five meshes, denoted by M3 to M7, where each mesh is derived from the previous one by a uniform refinement which approximately doubles the number of elements. We start from a base mesh M0, which is depicted in Fig.2. The numerical parameters of the meshes are summarized in Table 1.

In order to judge whether we have obtained a steady state we monitor the maximum value of $c_{x x}$ in the domain and the drag on the cylinder as a function of time. The time needed to obtain a steady state depends on the Weissenberg number Wi, but for higher Wi we need at least to compute until time $t>30$. For very high Wi the relaxation time becomes more important and at least several relaxation times must be computed before a reasonable steady state is obtained.

\subsection{Oldroyd-B model}

\subsubsection{Criterion for numerical instability}

In this section we try to verify that the criterion Eq. (24) determines the onset of the numerical instability in the flow around a cylinder for the standard FEM implementation. The breakdown of the solution sets in on the center line downstream of the cylinder close to the stagnation point. Therefore, we use 
the 1D expression for $C$ Eq. (32) on the center line. By breakdown we mean that we cannot reach a steady state within a certain time (we used $t=36$ ) and the solution 'explodes', that is the solution grows exponentially and reaches numerical overflow within a small number of time steps.

We will use mesh M4 which breaks down for $\mathrm{Wi}=0.88$. The numerical divergence starts to set in for $t \approx 20$ when starting up from a zero stress state. In Fig. 3 we give the values of $C$ along the center line in the Gauss integration points. This plot has been derived from the velocity along the center line extracted from the FEM calculation. Therefore the Gauss points are not real Gauss points in the FEM calculation, because these are interior to the 2D elements. These 1D Gauss points are expected to be the most relevant though for the onset of the instability and avoids calculating $C$ in points where the velocity is zero. In Fig. 3 we see that for $\mathrm{Wi}=0.7$ the value of $C$ is positive, indicating exponential growth in space, but small $(C<0.25)$. For higher Wi the region with positive $C$ becomes larger and the value of $C$ grows. For $\mathrm{Wi}=0.86$ the value of $C$ is smaller than 2 . For $\mathrm{Wi}=0.87$ the value of $C$ in the first point is larger than 2 (slightly higher than 3 ). For $\mathrm{Wi}=0.88$ the value of $C$ for time $t=18$ in the first point is close to 5 and the solution diverges after that. We see the same behavior for the coarser mesh M3 (results not shown), except that the value of $C$ in the first point becomes larger than 2 $(C=2.7)$ for $\mathrm{Wi}=0.86$ and the numerical instability sets in for $\mathrm{Wi}=0.87$, slightly lower than for mesh M4. In Fig. 3 we have also plotted the result for the computation using matrix logarithms for $\mathrm{Wi}=1.0$ using mesh M4. The computations are stable. The values of $C$ are significantly larger than 2 in both points of the first element without showing any numerical instabilities.

It is difficult to show the critical value of $C$ to be exactly 2 here, because that is proved to be the value for a constant velocity and constant growth factor, which is far from true in the first element. Furthermore, the 1D analysis is only an indication for the FEM computation, which is 2D. Anyway, we believe that the above results support the conclusion that the numerical instability as discussed in Sec. 4 is at work here.

\subsubsection{Drag results}

In Table 2 we give the value for the steady state dimensionless drag coefficient $K$

$$
K=\frac{F_{x}}{\eta U R}
$$

with $F_{x}$ the drag on the cylinder, for various value of Wi using different meshes. The most extensive set is for M4. All results are obtained using matrix logarithms. We also show the results of Fan et al. [14], Caola et al. [15] and Owens et al. [16], which are all close to ours. We did not include the results 


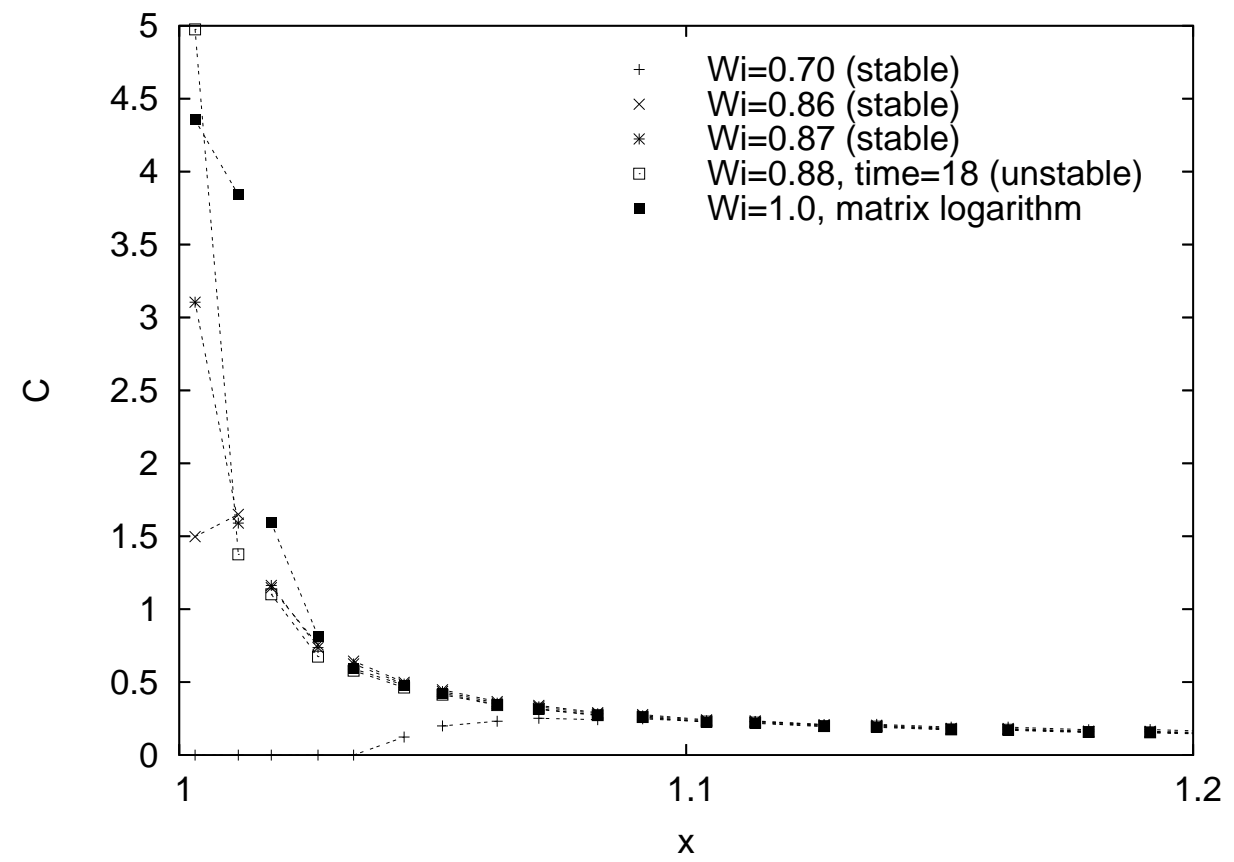

Fig. 3. The value of $C$ for various $\mathrm{Wi}$ on the centerline in the wake of the cylinder for the Oldroyd-B model. The mesh is M4. The values shown are in the (two) Gauss integration points and connected by a line.

of Alves et al. [17], which are close to the results of Fan et al. [14]. In contrast to the other authors we have no difficulty obtaining stable results beyond $\mathrm{Wi}=1.0$. It is clear that we have a nice convergence with mesh refinement. In the table we have indicated the onset of unsteady fluctuations by putting the result of $K$ between parentheses. The result printed is a value roughly before the fluctuations begin. The fluctuations in the drag are still small (say 0.1\%), but no steady state solution can be found. These fluctuations become worse for higher $\mathrm{Wi}$ and are likely to be numerical artefacts due to the incorrect stresses in the wake (see section 6.2.3). In Fig 4 we have also plotted the same results in a graph. It is clear that our results are very close to the results of Fan et al. [14], which are obtained using a higher-order FEM.

\subsubsection{Convergence of stresses}

The convergence of the drag coefficient with mesh refinement is not considered to be a very good indicator of accuracy. Therefore we have plotted in Fig. 5 for $\mathrm{Wi}=0.6$ and $\mathrm{Wi}=0.7$ the stress component $\tau_{x x}$ on the cylinder wall and along the center line in the wake. It is clear we have nice convergence for $\mathrm{Wi}=0.6$. For $\mathrm{Wi}=0.7$ we have more difficulty obtaining convergence, especially in the wake. For $\mathrm{Wi}=0.7$ we have also plotted the results of Fan et al. [14] and we see that on the cylinder wall we have the same maximum but our results are shifted slightly upstream. In the wake we seem to converge to higher values 
Table 2

Dimensionless steady state drag coefficient $K$

\begin{tabular}{ccccccccc}
\hline Wi & M3 & M4 & M5 & M6 & M7 & Ref. [14] & Ref. [15] & Ref. [16] \\
\hline 0.0 & & 132.358 & & & & 132.36 & 132.384 & 132.357 \\
0.1 & & 130.363 & & & & 130.36 & & \\
0.2 & & 126.626 & & & 126.62 & & \\
0.3 & & 123.193 & & & 123.19 & & \\
0.4 & & 120.596 & & & & 120.59 & & \\
0.5 & & 118.836 & & & & 118.83 & 118.763 & 118.827 \\
0.6 & 117.872 & 117.792 & 117.778 & 117.775 & 117.775 & 117.78 & & 117.775 \\
0.7 & 117.448 & 117.340 & 117.320 & 117.315 & 117.315 & 117.32 & & 117.291 \\
0.8 & & 117.373 & & & & 117.36 & & 117.237 \\
0.9 & & 117.787 & & & & 117.80 & & 117.503 \\
1.0 & 118.675 & 118.501 & 118.471 & & & 118.49 & 117.783 & 118.030 \\
1.1 & & 119.466 & & & & & 118.031 & 118.786 \\
1.2 & 120.860 & 120.650 & 120.613 & & & & & 119.764 \\
1.4 & 123.801 & 123.587 & $(123.541)$ & & & & & \\
1.6 & 127.356 & 127.172 & $(127.106)$ & & & & & \\
1.8 & $(131.458)$ & 131.285 & & & & & & \\
2.0 & & $(135.839)$ & & & & & & \\
\hline
\end{tabular}

than Fan et al. [14]. In the same figure we have also plotted the results for a one-dimensional DG calculation, starting from the back stagnation point, using the velocity component $v_{x}$ from the FEM calculation. The $1 \mathrm{D}$ calculation uses a very refined equidistant mesh with roughly 10 elements in the first element of the FEM solution. For $\mathrm{Wi}=0.6$ the results of the $1 \mathrm{D}$ calculation are consistent with the FEM results. For $\mathrm{Wi}=0.7$ the $1 \mathrm{D}$ results are higher and seems to be almost the same for mesh M4 and M5. This shows that we have not obtained convergence yet and that the converged result in the wake will likely be even higher than the results for our most refined mesh M7. The fact that the $1 \mathrm{D}$ results are roughly independent of the mesh indicates we have a stress convergence problem. This can be seen even more pronounced for $\mathrm{Wi}=1.0$. In Fig. 6 we have plotted $\tau_{x x}$ on the cylinder wall and along the center line in the wake again for $\mathrm{Wi}=1.0$. In the left figure we see a reasonable convergence on the cylinder surface, similar to $\mathrm{Wi}=0.7$, but no sign of convergence in the wake. If we do the $1 \mathrm{D}$ calculation here we see that the 'real' stresses are much higher than the results from the FEM and also mesh dependent. Therefore the FEM predictions of the stress in the wake are clearly 


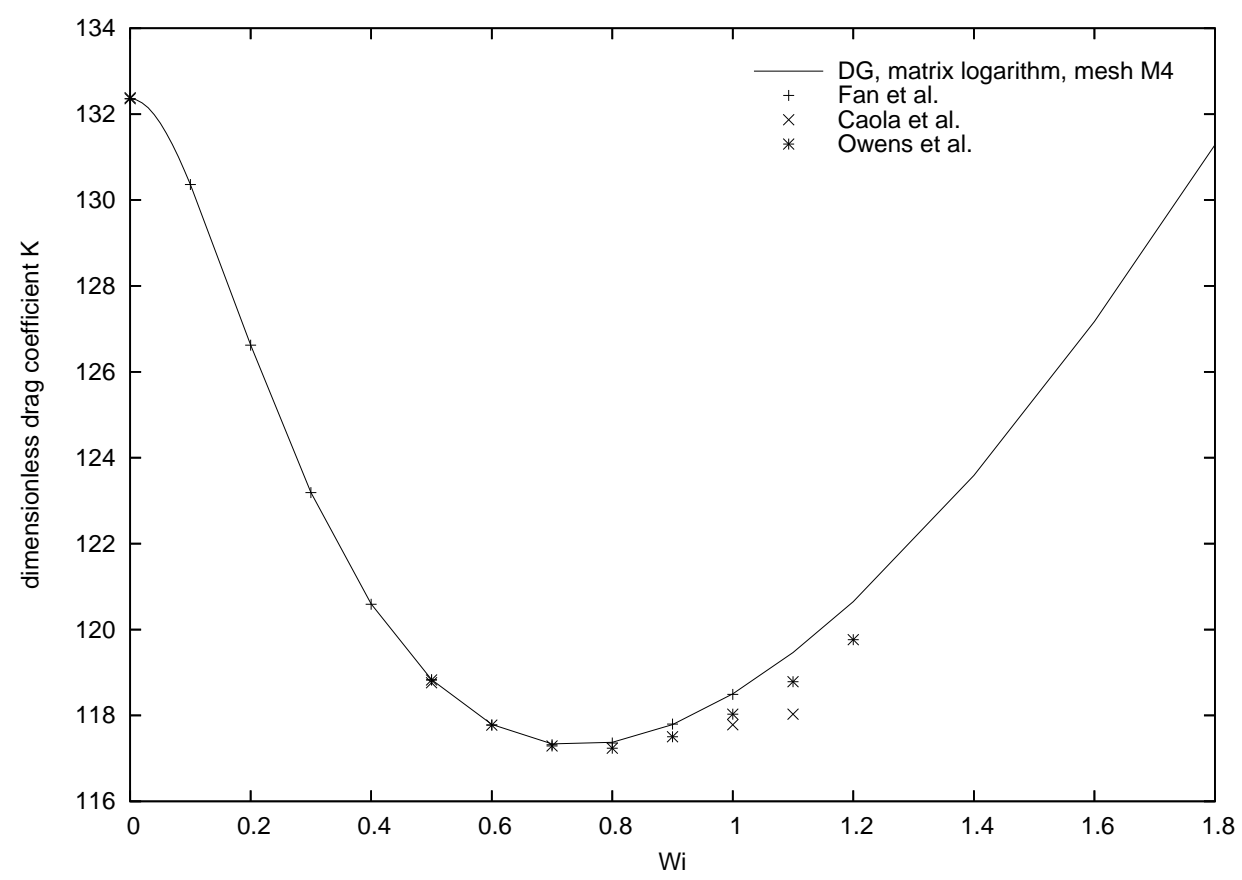

Fig. 4. Dimensionless drag $K$ versus Weissenberg number Wi for the flow past a cylinder in a channel using an Oldroyd-B model. The results of Alves et al. [17] are graphically close to the results of Fan et al. [14].

wrong. A possible reason is that the width of the stress wake is much smaller than the width of the elements in the wake. This is illustrated in Fig. 7, where it is clear that the width of the wake is small and becomes smaller with mesh refinement. Another 'proof' that the stresses on the centerline are incorrect is a plot of $\lambda \dot{\varepsilon}$, with $\dot{\varepsilon}=\partial v_{x} / \partial x$, on the centerline for $\mathrm{Wi}=1.0$ as depicted in Fig. 8. A value larger than 0.5 means exponential growth. If we take a closer look at the left figure in Fig. 6 we see that the stress maximum is near $x=1.4$ and decreasing with mesh refinement whereas $\lambda \dot{\varepsilon}>0.5$. This is inconsistent with a steady solution, since at the stress maximum the convection term is zero and exponential stretching would lead to an unsteady solution. The stress maximum in the $1 \mathrm{D}$ solution is at the point where $\lambda \dot{\varepsilon}=0.5$ near $x=1.9$. It is also clear from Fig. 8 that compared with the lower $\mathrm{Wi}=0.7$ the length scale over which the velocity gradient changes near the stagnation point is much smaller and this length scale is far from resolved for the most refined mesh (M5). It is not even clear whether a smooth solution near the cylinder exists! Nevertheless, the drag on the cylinder (see Table 2) seems to be unaffected by the convergence problems. This confirms the experience by many authors that the drag is a poor indicator of accuracy of the solution.

We should note here also that the maximum value of $c_{x x}$ in the wake for the $1 \mathrm{D}$ calculation using mesh M5 is almost 25000, which is in the range what can reasonably be expected as maximum stretch in real dilute polymer solutions. For example, in [18] the PS/PS dilute solution (a Boger fluid) has a FENE- 

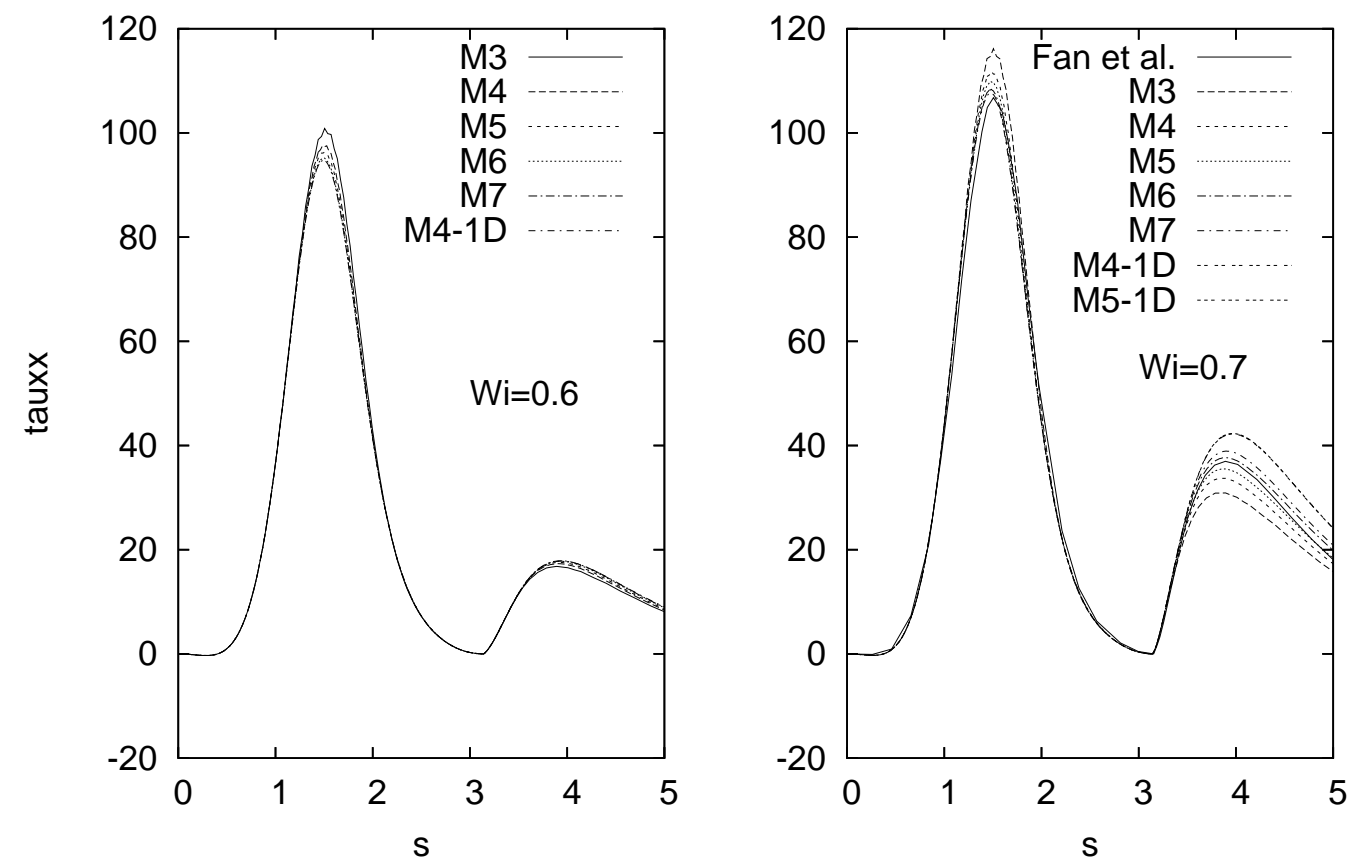

Fig. 5. The stress component $\tau_{x x}$ as a function of the curve coordinate $s$ along the cylinder surface and the center line in the wake of the cylinder. In the front stagnation point $s=0$ and at the back stagnation point $s=\pi$. Left figure: $\mathrm{Wi}=0.6$, right figure: $\mathrm{Wi}=0.7$.

$\mathrm{P}$ parameter $b$ of 26900 . This means that if the $1 \mathrm{D}$ result is any indication of the converged solution, $\mathrm{Wi}=1.0$ is clearly near the physical limit of the Oldroyd-B model for prediction of the stresses in the wake of real polymer solutions.

\subsubsection{Behavior at higher Wi}

Although the stresses in the wake are already incorrect at $\mathrm{Wi}=1.0$, the matrix logarithm method allows us to obtain stable numerical solutions for higher Wi. It is useful to describe the behavior of these solutions, because failure is quite different than what we were used to before, which usually was catastrophic failure. With the matrix logarithm the solution becomes unsteady at some Wi, depending on the mesh. For example, in Fig. 9 we show the stress profiles for two different Weissenberg numbers. For $\mathrm{Wi}=1.4$ the wake becomes unsteady for mesh M5 (not for M3 and M4). For larger times this shows up as a nonsmooth stress profile, whereas for smaller times it looks still smooth. The numerical solution however does not fail, in the sense that computations can be continued without a problem. At Wi $=1.6$ the numerical solution for mesh M5 is unsteady as well, but the stress profile remains smooth, as can be seen in the right figure of Fig. 9. For higher values of Wi the numerical solution becomes worse, also in other parts of the region, and eventually exponential 

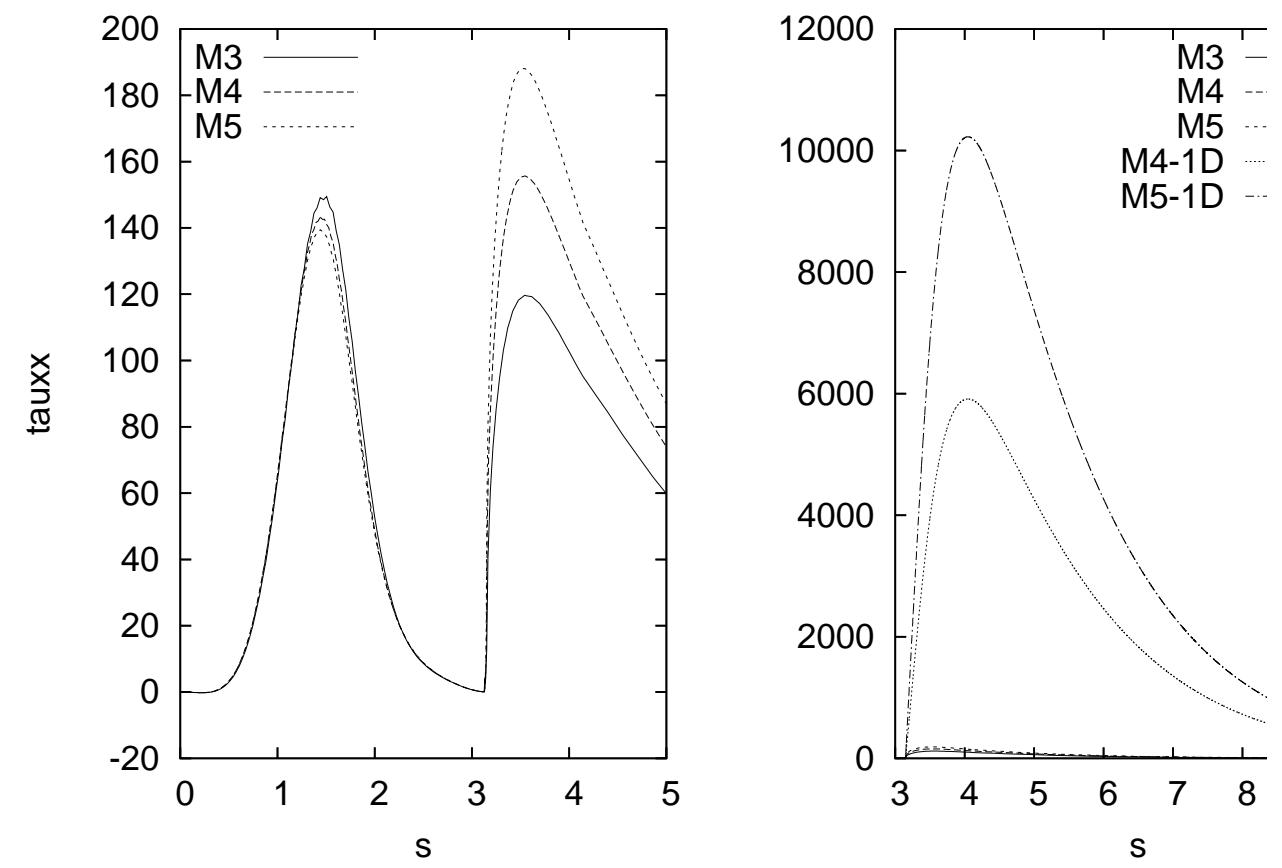

Fig. 6. The stress component $\tau_{x x}$ as a function of the curve coordinate $s$ along the cylinder surface and the center line in the wake of the cylinder for $\mathrm{Wi}=1.0$. In the front stagnation point $s=0$ and at the back stagnation point $s=\pi$. Left figure: FEM results, right figure: FEM results and 1D results in the wake. Note the different scales on the axes.

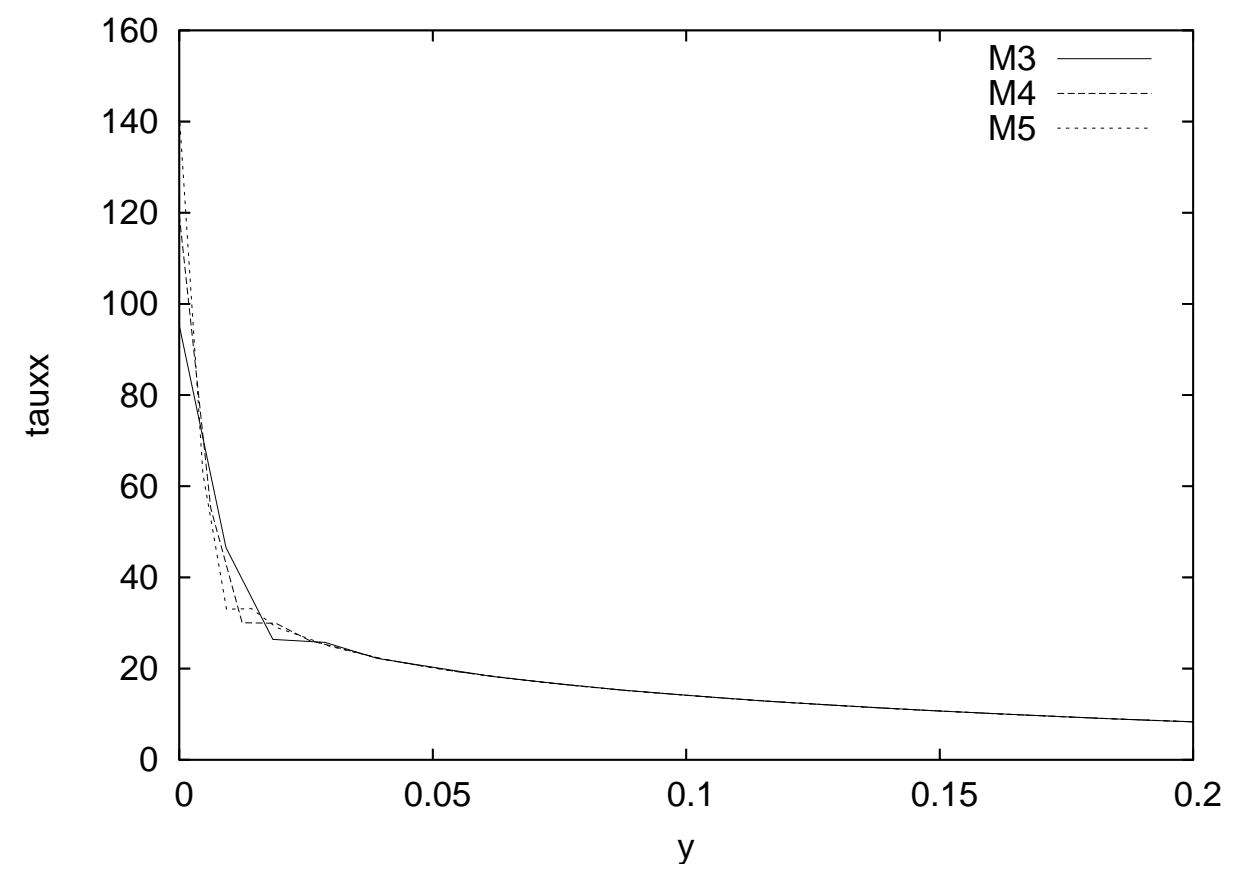

Fig. 7. The stress component $\tau_{x x}$ as a function of $y$ at the cross section $x=2$ for $\mathrm{Wi}=1.0$. Note the scale on the horizontal $(y)$ axis. 


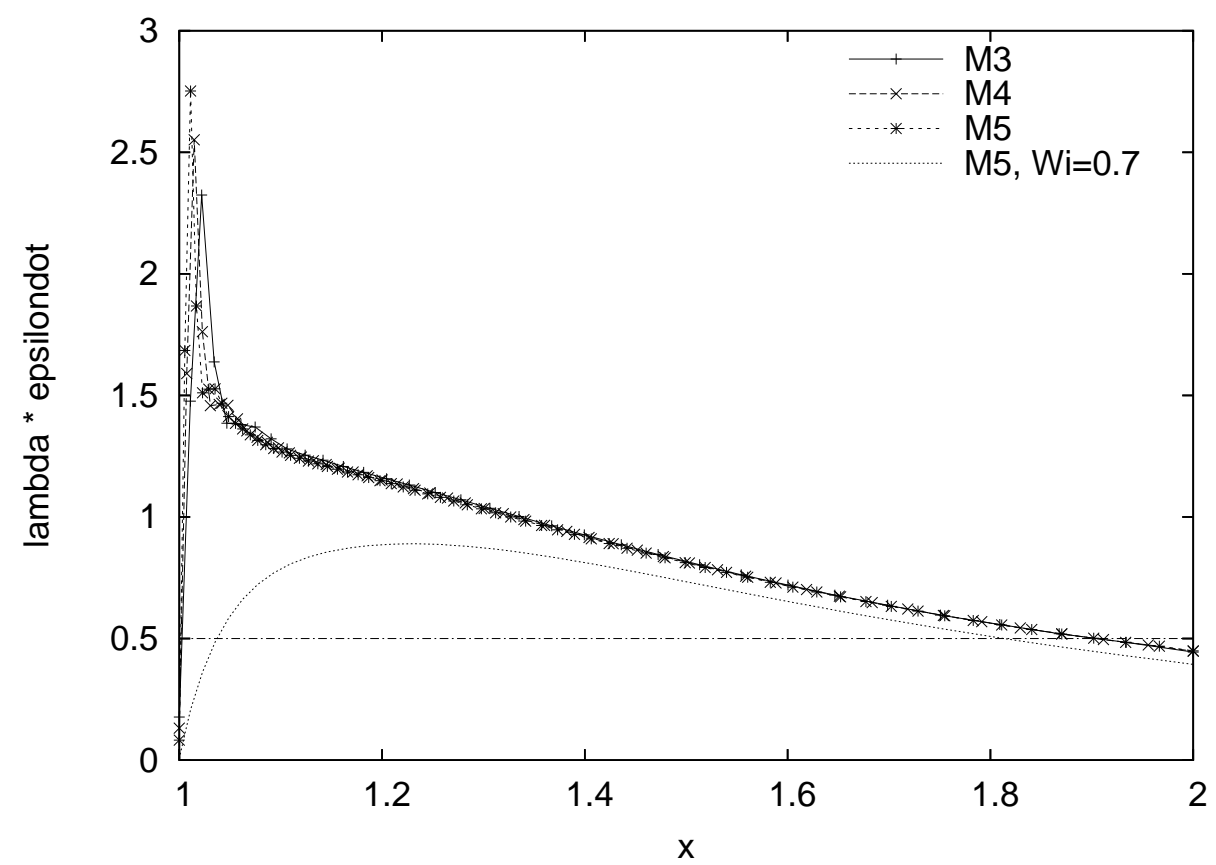

Fig. 8. The value of $\lambda \dot{\varepsilon}$ as a function of the coordinate $x$ on the center line in the wake of the cylinder for $\mathrm{Wi}=1.0$. Also shown is the result for $\mathrm{Wi}=0.7$ for one mesh.

growth sets in and no solution for larger times can be found anymore.

The remarkably better stability behavior of the matrix logarithm method for higher Wi can be underlined by examining the value of det $\boldsymbol{c}$. In previous methods the value of $\operatorname{det} \boldsymbol{c}$ becomes negative in a few points in the mesh at some rather low value $\mathrm{Wi}$ and is a precursor of the usual catastrophic instability for a slightly higher value of Wi. In Fig. 10 we show the value of $\log (\operatorname{det} \boldsymbol{c})=\operatorname{tr}(\log \boldsymbol{c})=\operatorname{tr} \boldsymbol{s}$ as a function of $x$ on the center line and on the cylinder wall for $\mathrm{Wi}=1.8$ with mesh M4. The value is larger than 0 , which means that $\operatorname{det} \boldsymbol{c}>1$. The latter is true in the complete region of the flow. Note, that det $\boldsymbol{c} \geq 1$ can be derived analytically for the Oldroyd-B model (see Hulsen [19]).

\subsection{Giesekus model}

The Oldroyd-B model is not a good model for high stretching, because the stretch (actually the conformation tensor $\boldsymbol{c}$ ) can grow to infinity even for a relatively small finite stretch rate. This is possibly causing the difficulties in the wake of the cylinder for the Oldroyd-B model. In order to limit the stretch to physical levels, nonlinear models must be used. For dilute polymer solutions the FENE type models are used, where the stretch is retricted to some finite value. For polymer melts and concentrated polymer solutions other 

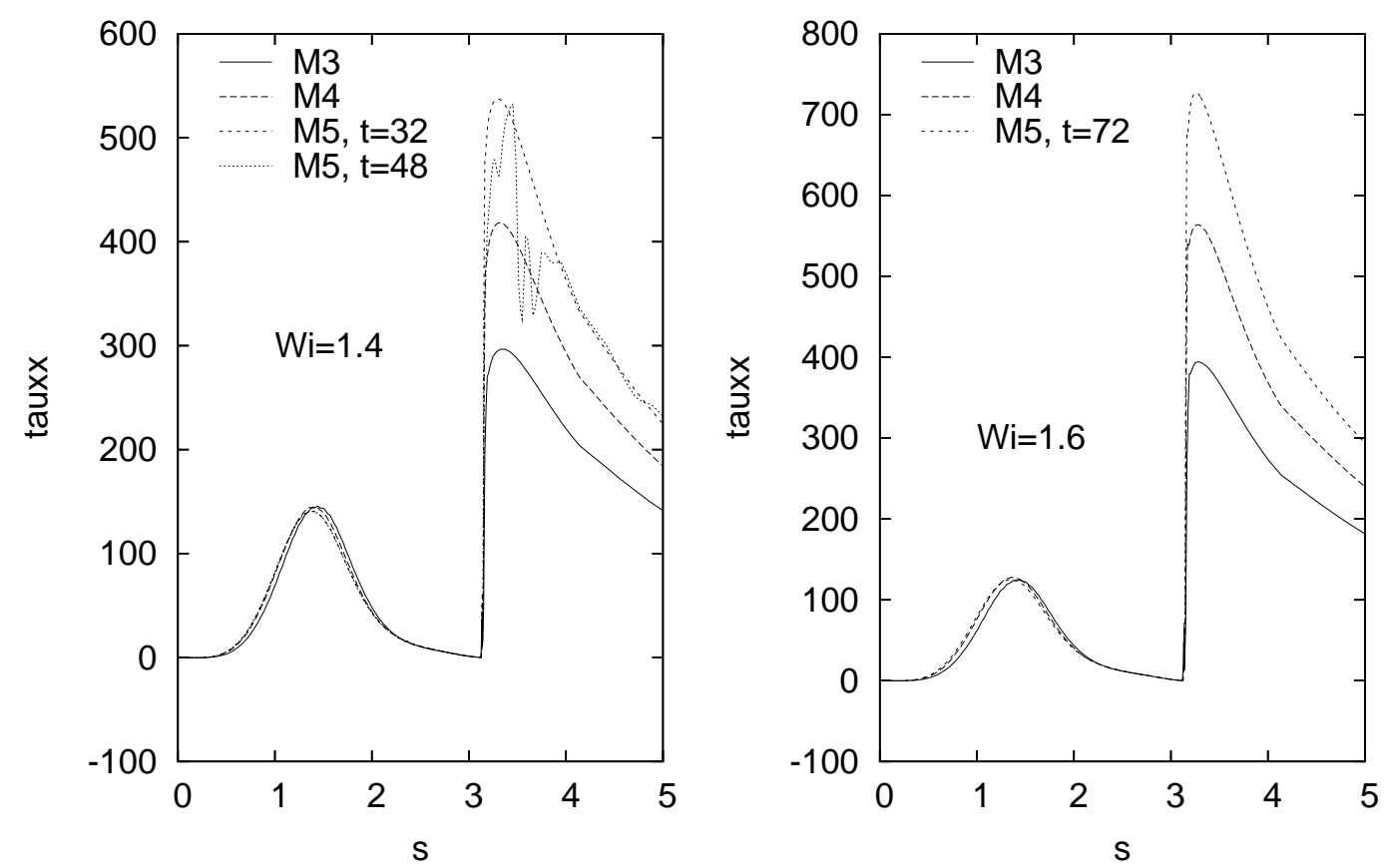

Fig. 9. The stress component $\tau_{x x}$ as a function of the curve coordinate $s$ along the cylinder surface and the center line in the wake of the cylinder. In the front stagnation point $s=0$ and at the back stagnation point $s=\pi$. Left figure: $\mathrm{Wi}=1.4$, right figure: $\mathrm{Wi}=1.6$.

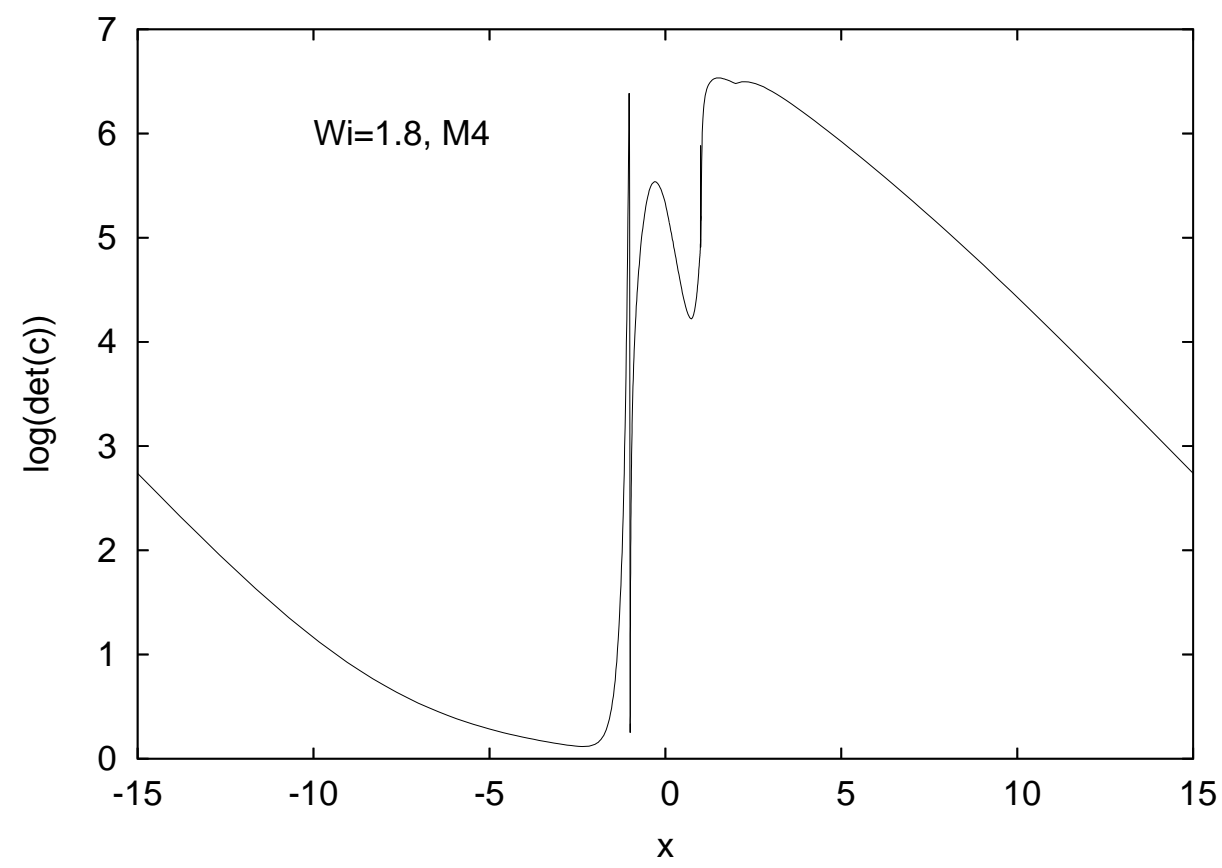

Fig. 10. The value of $\log (\operatorname{det} \boldsymbol{c})$ as a function of the coordinate $x$ on the center line in front of the cylinder, along the cylinder surface, and the center line in the wake of the cylinder. 
types of nonlinearity are introduced, such as the tube model (Doi-Edwards model) or anisotropic friction (Giesekus model). In this paper we will use the Giesekus model, because it is easy to implement and has all the ingredients to limit the stretch to show the real strength of the matrix logarithm method. It should be noted that for the Giesekus model the conformation tensor $\boldsymbol{c}$ is not limited to some finite value, but in order to reach infinity, the stretch rates must be infinite as well. We will choose a value of $\alpha=0.01$. This gives a two-dimensional Trouton ratio of $1 /(2 \alpha)=50$, still leading to subtantial strain-hardening, but compared to the Oldroyd-B the stretch is much more restricted. For polymer melts a larger value, for example $\alpha=0.25$ as in [7], with even more restricted strain-hardening seems to be more appropriate.

\subsubsection{Criterion for numerical instability}

Again we try to verify that the criterion Eq. (24) determines the onset of the numerical instability in the flow around a cylinder for the standard FEM implementation. We again check the 1D criterion Eq. (32) on the center line. Now the term in Eq. (32) involving $c_{x x}$ is non-zero. We will use a Giesekus model with $\alpha=0.01$. We will use mesh M3 which breaks down for $\mathrm{Wi}=1.20$, slightly higher than for the Oldroyd-B model (Wi = 0.87). In Fig. 11 we give the values of $C$ along the center line in the Gauss integration points. The contribution to $C$ of the extra term involving $c_{x x}$ is about $25 \%$ in the first element. We see that the behavior is similar to that obtained with the Oldroyd-B, except that we have a slightly higher Weissenberg number Wi now. For $\mathrm{Wi}=1.17$ the value of $C$ is smaller than 2 . For $\mathrm{Wi}=1.18$ and $\mathrm{Wi}=1.19$ the value of $C$ in the first point is larger than 2 (near 2.3 and 3.1, respectively). For $\mathrm{Wi}=1.20$ the solution breaks down. This again confirms our hypothesis that the numerical instability as discussed in Sec. 4 is the reason for numerical breakdown.

\subsubsection{Behavior at high Wi}

The behavior for high values of Wi of the Giesekus model with $\alpha=0.01$ using the FEM implementation with matrix logarithm is dramatically different than for the Oldroyd-B model: there does not seem to be a limit in Wi. In Fig. 12 we have plotted the component of the conformation tensor $c_{x x}$ on the cylinder wall and along the center line as a function of $x$ over the whole computed region for $\mathrm{Wi}=100$ for mesh M3 and M4. No convergence has been achieved just behind the cylinder in the wake and on the cylinder surface for these meshes. Note that for this high value of $\mathrm{Wi}$, the wake extends to the next cylinder in the periodic domain. Note also that the values of $c_{x x}$ are very high and that in the Giesekus model the nonlinear terms are two orders of magnitude larger than the linear terms near the maximum. In Fig. 13 the drag on the cylinder and 


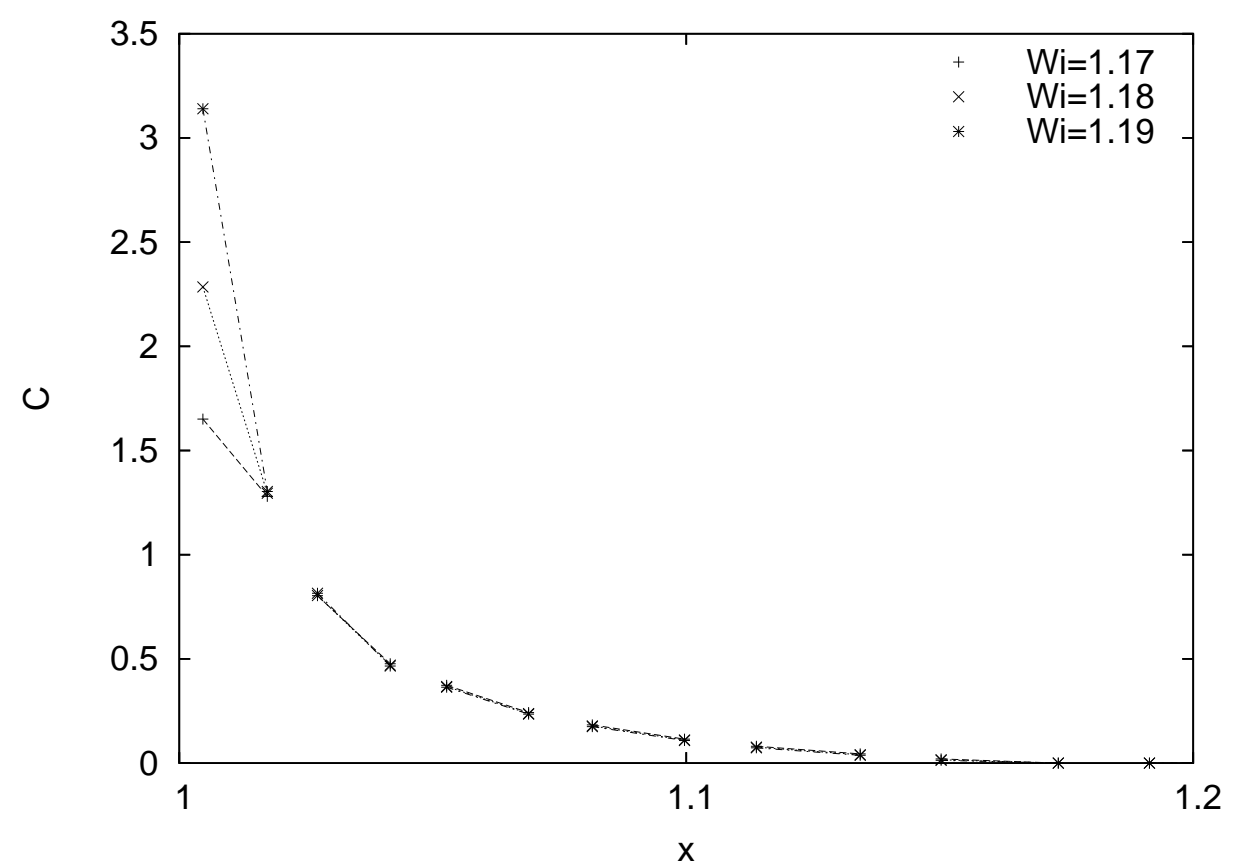

Fig. 11. The value of $C$ for various $\mathrm{Wi}$ on the centerline in the wake of the cylinder for the Giesekus model. The mesh is M3. The values shown are in the (two) Gauss integration points and connected by a line.

the maximum value of $c_{x x}$ in the flow is shown as a function of time for mesh M4. It is clear that two time scales seems to be acting here at the same time. The drag, which is mainly determined by the shear stresses on the cylinder, evolves in the time frame of one relaxation time, whereas the maximum $c_{x x}$ seems to evolve in a shorter time scale related to flow deformation. Note, that the time it takes for a particle on the center line to return to the same position (no cylinder present) is 20 whereas the relaxation time is 100 .

\subsubsection{Mesh convergence}

In the previous section we saw that at the high $\mathrm{Wi}=100$ convergence problems appear at localized regions. In this section we will consider the convergence problems at a lower $\mathrm{Wi}=5.0$, where they appear in a somewhat larger region in the wake. The results are shown in Fig. 14. Convergence on the cylinder is easily obtained, however convergence in the wake up to about one radius from the cylinder is very difficult. In the same figure we have also plotted the results of a one-dimensional DG calculation as explained in Sec. 6.2.3. We see that in the wake, where we have convergence problems, the 1D calculation gives locally near the cylinder significantly higher values for $c_{x x}$, but surely not as dramatic as we saw for the Oldroyd-B problem. Also the typical length scale involved seems to be much smaller. This is an indication that for mesh convergence in this region we need at least a mesh that is much more refined 


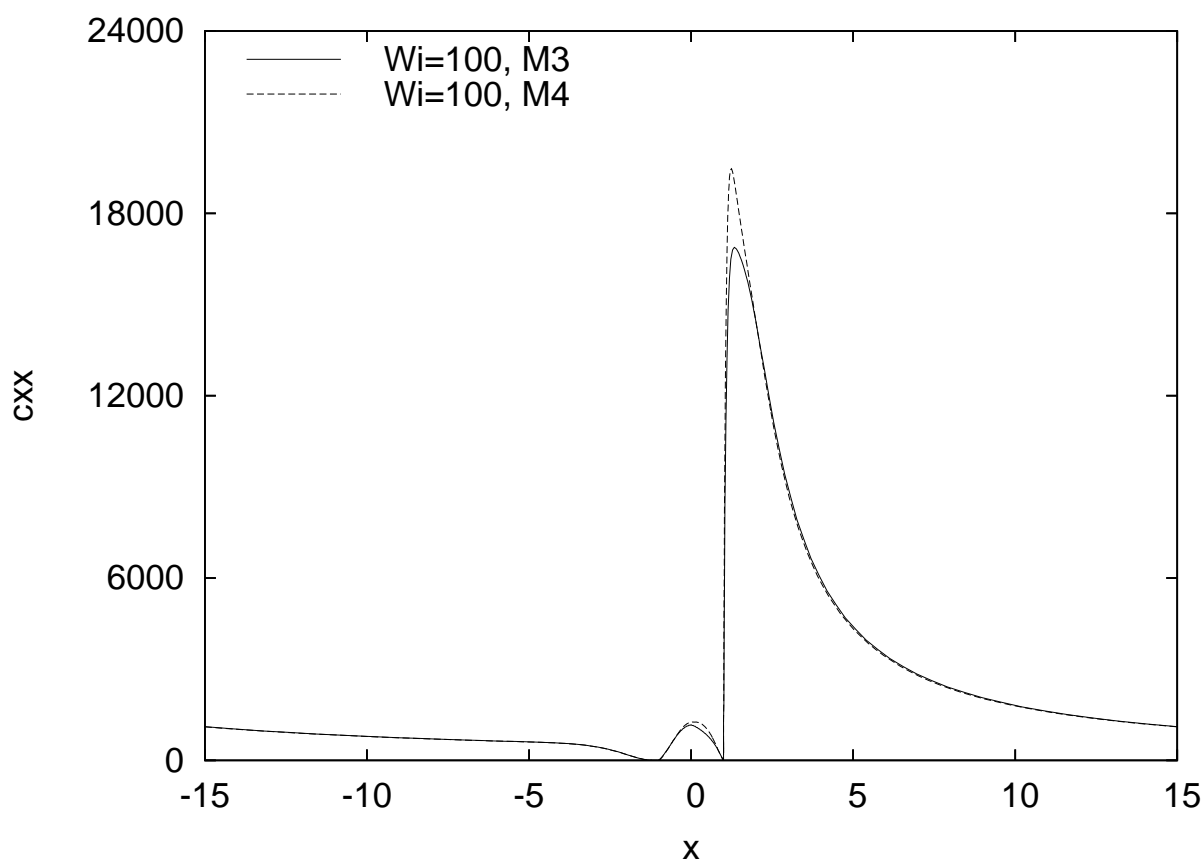

Fig. 12. The value of $c_{x x}$ for $\mathrm{Wi}=100$ on the centerline and on the wall of the cylinder for the Giesekus model with $\alpha=0.01$. Two meshes are shown: M3 and M4.
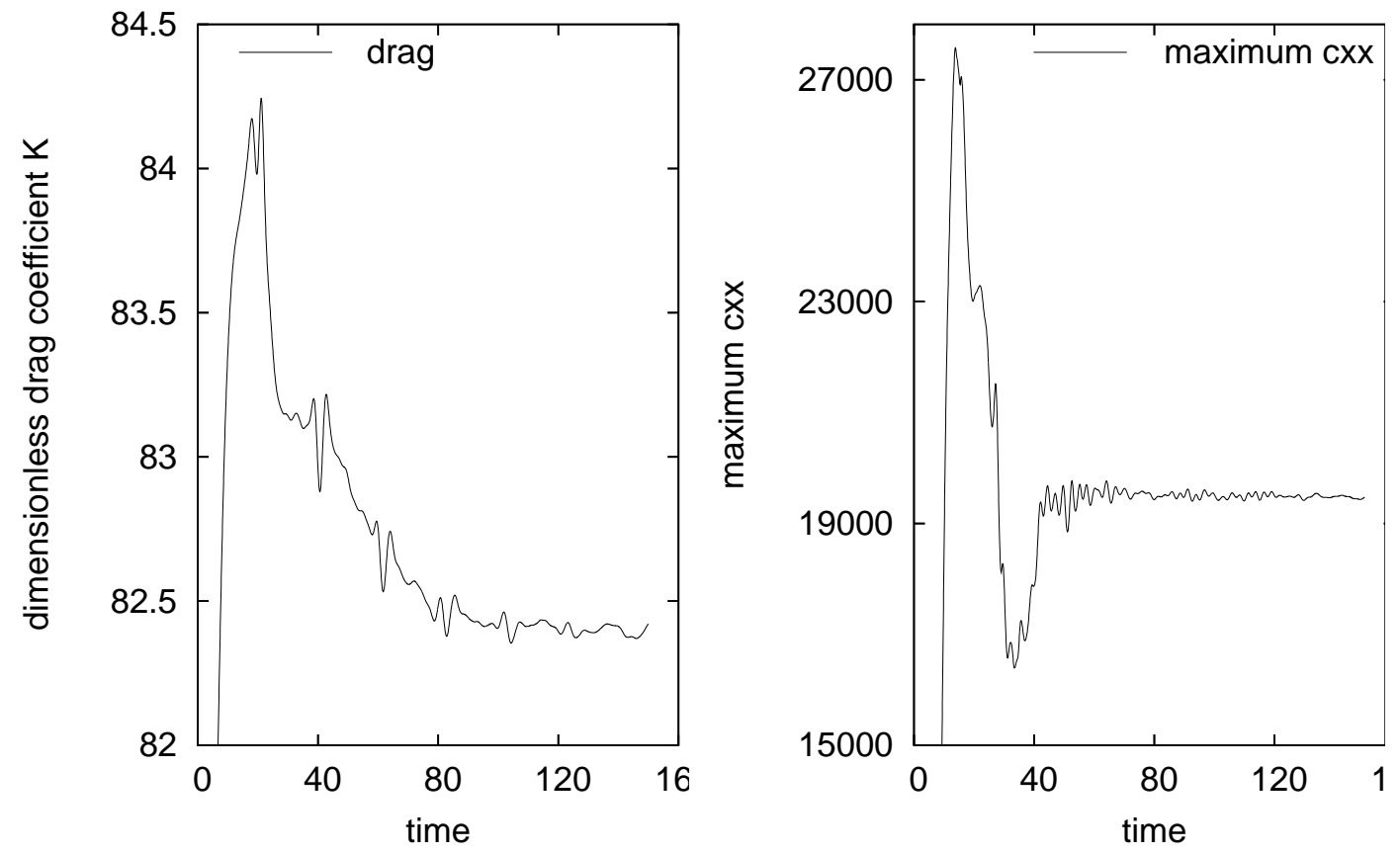

Fig. 13. The drag coefficient $K$ and the maximum value of $c_{x x}$ as a function of time for $\mathrm{Wi}=100$. the Giesekus model with $\alpha=0.01$. The mesh is M4. 


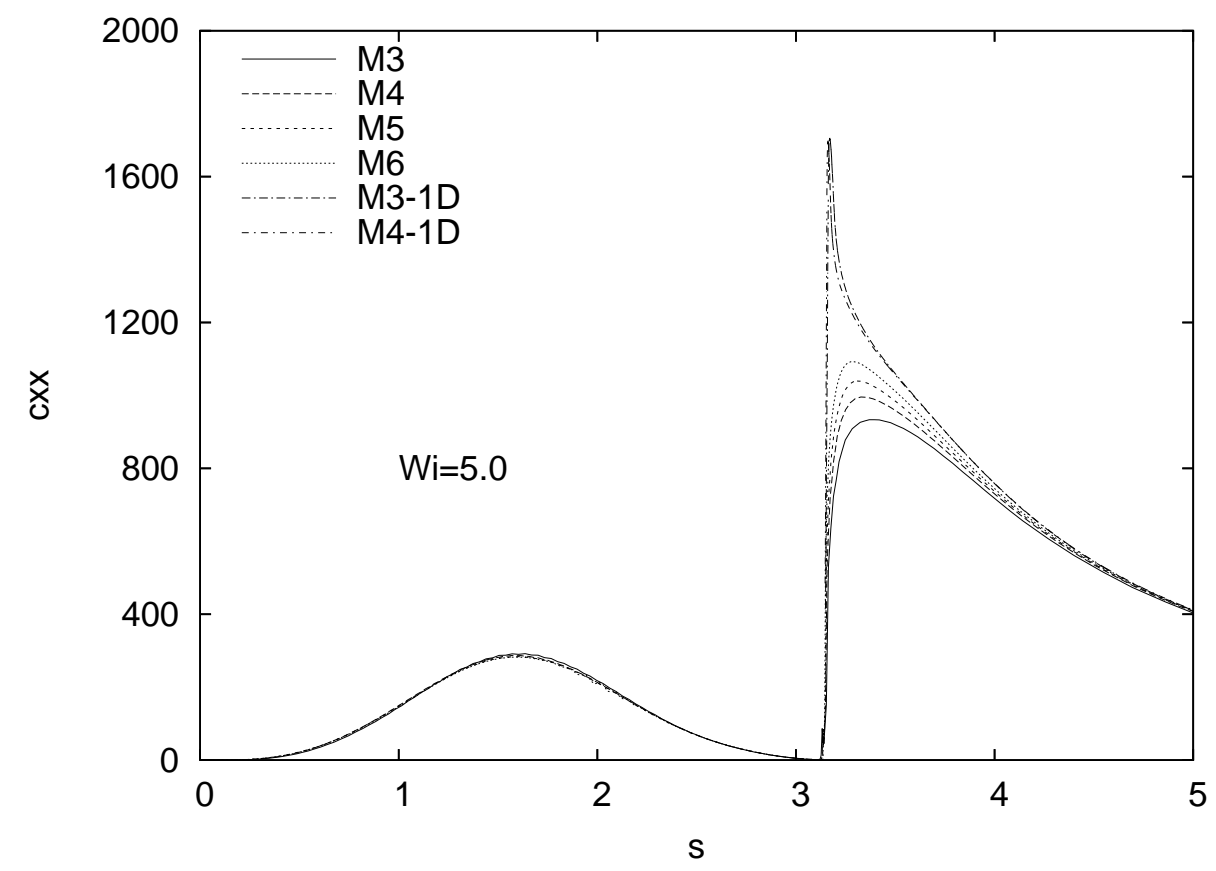

Fig. 14. The value of $c_{x x}$ for $\mathrm{Wi}=5$ on the centerline and on the wall of the cylinder for the Giesekus model with $\alpha=0.01$ for various meshes. Also shown are the results for meshes M3 and M4 using the 1D procedure as explained in Sec. 6.2.3.

than our most refined meshes. A way to achieve convergence is possibly by adaptive local refinement or higher-order methods, but problems of another nature, such as inproper discretization and model problems cannot be ruled out either. More work is needed here. However, this is beyond the scope of this paper.

\section{Conclusions and discussion}

It has been shown that also in the FEM implementation, the log conformation representation removes the catastrophic breakdown present in the standard FEM implementation. We used a standard benchmark problem: the flow around a cylinder. We provided some clear evidence that the breakdown can be attributed to the failure of the numerical solution to balance the exponential growth by the convection. So, in a way, we believe that the HWNP-problem has been solved. That doesn't mean all problems are solved.

Since we are able to obtain solutions now, we can judge the quality of these solutions. It turns out that high-Weissenberg problems remain notoriously difficult due to the exponential behavior and convergence problems appear. For the case of the flow around a cylinder for the Oldroyd-B model we believe these might be related to a model artefact, that is the unlimited extension of the 
polymer at finite extension rates. It is possible that no solutions exist beyond some Weissenberg number, but further investigations are needed to answer that question. For the Giesekus model, which significantly reduces the extension, there does not seem to be a limit to the obtainable Weissenberg numbers for the chosen parameters of the model. Like for the Oldroyd-B model, convergence problems in localized regions exist, in particular in the wake near the cylinder. However, for the Giesekus model they seem manageable and very refined local meshes and/or higher-order methods might be appropriate to obtain convergence in these localized regions as well. This is however beyond the scope of this paper and further work is needed to determine the precise reason of the convergence problems.

Another open question that remains is: suppose we have been able to obtain convergence at some high Weissenberg by some higher-order scheme with very refined meshes, will the standard method be stable also? After all the $C$ parameter (see Eq. (22)) should go to zero for infinite refinement for a smooth solution. Even if this turns out to be true in the end, the matrix log method proposed here has the advantage of having the ability to obtain solutions for relatively coarse meshes, which are accurate in large parts of the flow. The user can evaluate the solution and after some analysis might conclude that local inaccuracies are unimportant for the practical problem at hand. We consider this to be a huge improvement for practical problems.

\section{Acknowledgements}

We thank Frank Baaijens for stimulating discussions and for giving the first author the opportunity to present this work at the ICR2004.

This research was funded in part by the Applied Mathematical Sciences subprogram of the Office of Energy Research of the US Department of Energy under Contract DE-AC03-76-SF00098.

\section{References}

[1] R. Fattal and R. Kupferman. Constitutive laws for the matrix-logarithm of the conformation tensor. J. Non-Newtonian Fluid Mech., 2004. in press.

[2] R. Keunings. A survey of computational rheology. In D.M. Binding et al., editor, Proceedings of the XIIIth International Congress on Rheology, Cambridge, UK, volume 1, pages 7-14, Glasgow, UK, 2000. British Society of Rheology. 
[3] R. Fattal and R. Kupferman. Time-dependent simulation of viscoelastic flows at high weissenberg number using the log-conformation representation. J. NonNewtonian Fluid Mech., 2004. submitted.

[4] M.A. Hulsen, A.P.G. van Heel, and B.H.A.A. van den Brule. Simulation of viscoelastic flows using Brownian configuration fields. J. Non-Newtonian Fluid Mech., 70:79-101, 1997.

[5] R. Guénette and M. Fortin. A new mixed finite element method for computing viscoelastic flows. J. Non-Newtonian Fluid Mech., 60:27-52, 1995.

[6] M. Fortin and A. Fortin. A new approach for the FEM simulation of viscoelastic flows. J. Non-Newtonian Fluid Mech., 32:295-310, 1998.

[7] F.P.T. Baaijens, S.H.A. Selen, H.P.W. Baaijens, G.W.M. Peters, and H.E.H. Meijer. Viscoelastic flow past a confined cylinder of a low density polyethylene melt. J. Non-Newtonian Fluid Mech., 68:173-203, 1997.

[8] A. Fortin, R. Guénette, and R. Pierre. On the discrete EVSS method. Comp. Meth. Appl. Mech. Eng., 189:121-139, 2000.

[9] R. LeVeque. Numerical Methods for Conservation Laws. Birkhauser Verlag, Basel, 1992.

[10] M.A. Hulsen, E.A.J.F. Peters, and B.H.A.A. van den Brule. A new approach to the deformation fields method for solving complex flows using integral constitutive equations. J. Non-Newtonian Fluid Mech., 98:201-221, 2001.

[11] M.A. Hulsen. Analysis and Numerical Simulation of the Flow of Viscoelastic Fluids. PhD thesis, Delft University of Technology, Delft, The Netherlands, 1988.

[12] J. van der Zanden and M.A. Hulsen. Mathematical and physical requirements for successful computations with viscoelastic fluid models. J. Non-Newtonian Fluid Mech., 29:93-117, 1988.

[13] R. Hill. Aspect of invariance in solid mechanics. Advances in Applied Mechanics, 18:1-75, 1978.

[14] Y. Fan, R.I. Tanner, and N. Phan-Thien. Galerkin/least-square finite-element methods for steady viscoelastic flows. J. Non-Newtonian Fluid Mech., 84:233256, 1999 .

[15] A.E. Caola, Y.L. Joo, R.C. Armstrong, and R.A. Brown. Highly parallel time integration of viscoelastic flows. J. Non-Newtonian Fluid Mech., 100:191-216, 2001.

[16] R.G. Owens, C. Chauvière, and T.N. Philips. A locallly-upwinded spectral technique (LUST) for viscoelastic flows. J. Non-Newtonian Fluid Mech., 108:49$71,2002$.

[17] M.A. Alves, P.J. Oliviera, and F.T. Pinho. The flow of viscoelastic fluids past a cylinder: finite-volume high-resolution methods. J. Non-Newtonian Fluid Mech., 97:207-232, 2001. 
[18] J.P. Rothstein and G.H. McKinley. Inhomogeneous transient uniaxial extensional rheometry. J. Rheol., 46:1419-1443, 2002.

[19] M.A. Hulsen. Some properties and analytical expressions for plane flow of Leonov and Giesekus models. J. Non-Newtonian Fluid Mech., 30:85-92, 1988. 